\title{
A NOTE ON INTERPOLATION
}

\section{GEORGE KLEIN}

Let $f(x)$ be a function of period $2 \pi$ and let $I_{n}(x ; f)$ be the trigonometric polynomial of order $n$ coinciding with $f$ at $2 n+1$ equidistant points

$$
\begin{aligned}
\left\{x_{0}, x_{1}, \cdots, x_{2 n}\right\} & =\left\{x_{0}, x_{0}+h, \cdots, x_{0}+2 n h\right\}, \\
\cdot h & =h_{n}=\frac{2 \pi}{2 n+1},
\end{aligned}
$$

of the interval $[0,2 \pi]$. The familiar formula of Lagrange for $I_{n}(x ; f)$ can be written in the form

$$
I_{n}(x ; f)=\frac{1}{\pi} \int_{0}^{2 \pi} f(t) D_{n}(x-t) d \omega_{2 n+1}(t)
$$

where $D_{n}(u)=2^{-1} \sum_{v=-n}^{n} e^{i v u}$ is the Dirichlet kernel and $\omega_{2 n+1}(t)$ is a step function constant in the interior of the intervals $\left[x_{0}+k h\right.$, $\left.x_{0}+(k+1) h\right]$ and having a jump $h$ at the points $x_{0}+k h .^{1}$ Thus $\omega_{2 n+1}(t)$ is determined up to an arbitrary additive constant. It is well known that $I_{n}(x ; f)$ need not tend to $f(x)$ even if $f$ is everywhere continuous.

S. Bernstein ${ }^{2}$ pointed out that the situation is considerably improved if we consider the trigonometric polynomial of order $n$ assuming at the points $x_{k}$ the values

$$
2^{-1}\left\{f\left(x_{k}-h / 2\right)+f\left(x_{k}+h / 2\right)\right\} \quad(k=0,1, \cdots, 2 n) .
$$

In this case, for every bounded $f$, the interpolating polynomials are contained between the upper and lower bounds of $f$ and converge to $f$ at every point of continuity, the convergence being uniform over every closed interval of continuity. The same holds if we take the polynomials assuming at the points $x_{k}$ the values

$$
2^{-2}\left\{f\left(x_{k}-h\right)+2 f\left(x_{k}\right)+f\left(x_{k}+h\right)\right\}
$$

or

(5) $2^{-3}\left\{f\left(x_{k}-3 h / 2\right)+3 f\left(x_{k}-h / 2\right)+3 f\left(x_{k}+h / 2\right)+f\left(x_{k}+3 h / 2\right)\right\}$

Received by the editors June 21, 1949.

1 This notation seems to have first been used by J. Marcinkiewicz, On interpolation polynomials (in Polish), Wiadomosci Matematyczne vol. 39 (1935) pp. 207-221.

$2 \mathrm{~S}$. Bernstein, Sur une modification de la formule d'interpolation de Lagrange, Communications of the Mathematical Society of Kharkov vol. 5 pp. 49-57. 
$(k=0,1, \cdots, 2 n)$, and so on.

It is natural to expect that in the cases (4), (5), and so on, the convergence of the interpolating polynomials to $f$ is stronger than is (3), and we shall show that this is so by proving that then even the differentiated polynomials converge to the derivatives of $f$, provided the latter exist (a fact which has practical uses). More precisely, our result is as follows:

TheOREM. Let $p$ be a positive integer and let $f$ be a bounded measurable function of period $2 \pi$ which has at the point $x^{\prime}$ a derivative of order $j<p$. Let $T_{n, p}(x ; f)$ be the trigonometric polynomial of order $n$ which assumes at the points $x_{k}=x_{0}+k h\left(k=0,1, \cdots, 2 n ; h=h_{n}=2 \pi /(2 n+1)\right)$ the values

(6) $f_{k}=\frac{1}{2^{p}} \sum_{i=0}^{p} C_{p, i} f\left(x_{k}+\left(i-\frac{p-1}{2}\right) h\right)$, where $C_{p, i}=\frac{p !}{i !(p-i) !}$

Then

$$
\lim _{n \rightarrow \infty} \frac{d^{i}}{d x^{j}} T_{n, p}\left(x^{\prime} ; f\right)=f^{(j)}\left(x^{\prime}\right) .
$$

In this theorem the number $x_{0}=x_{0}^{(n)}$ may depend on $n$. The derivative here is taken in the generalized sense due to Peano and de la Vallee-Poussin, that is, if for small values of $|t|$,

$$
f(x+t)=a_{0}+\frac{a_{1}}{1 !} t+\frac{a_{2}}{2 !} t^{2}+\cdots+\frac{a_{j-1}}{(j-1) !} t^{j-1}+\frac{\left(a_{j}+\epsilon_{t}\right)}{j !} t^{j}
$$

where the $a$ 's are constants and $\epsilon_{t} \rightarrow 0$ as $t \rightarrow 0$, then $f$ is said to have a $j$ th generalized derivative at $x$, which is equal to $a_{j}$.

Using (2) one easily sees that

$$
\begin{aligned}
T_{n, p}(x ; f) & \\
= & \frac{1}{2^{p}} \sum_{j=0}^{p} C_{p, j} \frac{1}{\pi} \int_{0}^{2 \pi} f\left(t+\left(j-\frac{p-1}{2}\right) h\right) D_{n}(x-t) d \omega_{2 n+1}(t) \\
= & \frac{1}{2^{p}} \sum_{j=0}^{p} C_{p, j} \frac{1}{\pi} \int_{0}^{2 \pi} f(t) D_{n}\left(x+\left(j-\frac{p-1}{2}\right) h-t\right) \\
& \cdot d \omega_{2 n+1}\left(t-\left(j-\frac{p-1}{2}\right) h\right) .
\end{aligned}
$$

If $p$ is odd, then $d \omega_{2 n+1}(t-(j-(p-1) / 2) h)$ may be replaced by $d \omega_{2 n+1}(t)$, and if $p$ is even,-by $d \omega_{2 n+1}(t+h / 2)$. Setting, therefore, 


$$
\omega_{2 n+1}^{*}(t)=\omega_{2 n+1}(t) \quad \text { if } \quad p \text { is odd }
$$

and

$$
\omega_{2 n+1}^{*}(t)=\omega_{2 n+1}(t+h / 2) \quad \text { if } p \text { is even, }
$$

we see that

$$
T_{n, p}(x ; f)=\frac{1}{\pi} \int_{0}^{2 \pi} f(t) D_{n, p}(x-t) d \omega_{2 n+1}^{*}(t)
$$

where

$$
\begin{aligned}
D_{n, p}(u) & =\frac{1}{2^{p}} \sum_{j=0}^{p} C_{p, j} D_{n}\left(u+\left(j-\frac{p-1}{2}\right) h\right) \\
& =\frac{1}{2^{p}} \sum_{j=0}^{p} C_{p, j} \frac{1}{2} \sum_{\nu=-n}^{n} e^{i \nu(u+(j-(p-1) / 2) h)} \\
& =\frac{1}{2} \sum_{\nu=-n}^{n} e^{i \nu u} \frac{1}{2^{p}} \sum_{j=0}^{p} C_{p, j} e^{i(j-(p-1) / 2) \nu h} \\
& =\frac{1}{2} \sum_{\nu=-n}^{n} e^{i \nu u} \cos ^{p} \frac{\nu h}{2}=\frac{1}{2}+\sum_{\nu=1}^{n} \cos \nu u \cos ^{p} \frac{\nu h}{2} .
\end{aligned}
$$

In formula (8) we can write $d \omega_{2 n+1}(t)$ instead of $d \omega_{2 n+1}^{*}(t)$, since this amounts to translating $x_{0}$ by $h / 2$ for $p$ even, and as we have already stated our $x_{0}$ is arbitrary. If the $\nu$ th partial sum of the polynomial $I_{n}$ (see (2)) is denoted by $I_{n, v}$, we have

$$
I_{n, \nu}(x ; f)=\frac{1}{\pi} \int_{0}^{2 \pi} f(t) D_{\nu}(x-t) d \omega_{2 n+1}(t) .
$$

Let us apply summation by parts to the last sum in (9). We get

$$
D_{n, p}(u)=\sum_{\nu=0}^{n-1} D_{\nu}(u) \Delta \cos ^{p} \frac{\nu h}{2}+D_{n}(u) \cos ^{p} \frac{n h}{2},
$$

and this inserted in (8) gives (see (10))

$$
T_{n, p}(x ; f)=\sum_{\nu=0}^{n-1} I_{n, \nu}(x ; f) \Delta \cos ^{p} \frac{\nu h}{2}+I_{n}(x ; f) \cos ^{p} \frac{n h}{2} .
$$

For any sequence $a_{0}, a_{1}, \cdots$, we use the notation $\Delta a_{\nu}=a_{\nu}-a_{\nu+1}$, $\Delta^{*} a=\Delta\left(\Delta^{s-1} a_{v}\right)$.

Let $S_{n, \nu}^{k}$ and $I_{n, \nu}^{k}$ denote the $k$ th Cesaro sums and the $k$ th Cesaro means, respectively, of the sequence $I_{n, \nu}$ for $n$ fixed. Thus 


$$
\begin{gathered}
S_{n, \nu}^{0}=I_{n, \nu}, S_{n, \nu}^{k}=S_{n, 0}^{k-1}+S_{n, 1}^{k-1}+\cdots+S_{n, \nu}^{k-1}, \\
I_{n, \nu}^{k}=\frac{S_{n, \nu}^{k}}{A_{\nu}^{k}}, \text { with } A_{\nu}^{k}=C_{v+k, v}
\end{gathered}
$$

Applying to the right side of (11) repeated summation by parts we easily get

$$
\begin{aligned}
T_{n, p}(x ; f)= & \sum_{\nu=0}^{n-2} S_{n, \nu}^{1}(x ; f) \Delta^{2} \cos ^{p} \frac{\nu h}{2}+S_{n, n-1}^{1}(x ; f) \Delta \cos ^{p} \frac{(n-1) h}{2} \\
& +S_{n, n}^{0} \cos ^{p} \frac{n h}{2} \\
= & \ldots \ldots \ldots \ldots \ldots \ldots \ldots \ldots \\
= & \sum_{\nu=0}^{n-p-1} S_{n, \nu}^{p}(x ; f) \Delta^{p+1} \cos ^{p} \frac{\nu h}{2} \\
& +\sum_{k=0}^{p} S_{n, n-p+k}^{p-k}(x ; f) \Delta^{p-k} \cos ^{p} \frac{(n-p+k) h}{2} \\
= & \sum_{\nu=0}^{n-p-1} I_{n, \nu}^{p}(x ; f) A_{\nu}^{p} \Delta^{p+1} \cos ^{p} \frac{\nu h}{2} \\
& +\sum_{k=0}^{p} I_{n, n-p+k}^{p-k}(x ; f) A_{n-p+k}^{p-k} \Delta^{p-k} \cos ^{p} \frac{(n-p+k) h}{2} .
\end{aligned}
$$

Hence

$$
\begin{aligned}
& \frac{d^{j}}{d x^{j}} T_{n, p}\left(x^{\prime} ; f\right)=\sum_{p=0}^{n-p-1} \frac{d^{j}}{d x^{j}} I_{n, \nu}^{p}\left(x^{\prime} ; f\right) A_{, p}^{p} \Delta^{p+1} \cos ^{p} \frac{\nu h}{2} \\
&+\sum_{k=0}^{p} \frac{d^{j}}{d x^{i}} I_{n, n-p+k}^{p-k}\left(x^{\prime} ; f\right) A_{n-p+k}^{p-k} \Delta^{p-k} \cos ^{p} \frac{(n-p+k) h}{2} .
\end{aligned}
$$

By way of making clear our reasons for expressing $d^{i} T_{n, p}\left(x^{\prime} ; f\right) / d x^{j}$ in the form (12), we observe first that if $f$ happens to be a trigonometric polynomial $P$, then the theorem is true. For then, as seen from (6), $T_{n, p}(x ; f)=\left(1 / 2^{p}\right) \sum_{j=0}^{p} C_{p, j} P(x+(j-(p-1) / 2) h)$. Differentiating this equation $j$ times and letting $n \rightarrow \infty$, we get $T_{n, p}^{(j)}\left(x^{\prime} ; f\right) \rightarrow p^{(j)}\left(x^{\prime}\right)$. Furthermore we can find a polynomial $P$ which has the same derivatives as $f$ at the point $x^{\prime}$. Subtracting $P$ from $f$, we can suppose that $f\left(x^{\prime}+t\right)=o\left(|t|^{j}\right)$ if $f^{(j)}\left(x^{\prime}\right)$ exists. Our task is then to show that for such $f$ the expression (12) approaches zero as $n \rightarrow \infty$. 
To this end we shall evaluate the orders of the various terms on the right side of (12) for such $f$. In particular we shall show that

$$
\frac{d^{j}}{d x^{j}} I_{n, \nu}^{p}\left(x^{\prime} ; f\right) \rightarrow 0 \text { as } \nu \rightarrow \infty, \nu \leqq n, \text { for } j=0,1, \cdots, p-1 .
$$

Once (13) is established, it will immediately follow that

(14) $\frac{d^{i}}{d x^{i}} I_{n}^{p-{ }^{k}{ }^{k}}\left(x^{\prime} ; f\right)=o(\nu), k=0,1, \cdots, p ; j=0,1, \cdots, p-1$.

For $k=0,(14)$ is the assertion (13). We assume that (14) has been proved for $k=0,1, \cdots, k_{1}$. Then

$$
\begin{aligned}
\frac{d^{i}}{d x^{i}} I_{n, \nu}^{p-\left(k_{1}+1\right)}\left(x^{\prime} ; f\right) & =-\frac{1}{A_{\nu}^{p-(k 1+1)}} \Delta A_{\nu-1}^{p-k_{1}} \frac{d^{i}}{d x^{i}} I_{n, \nu-1}^{p-k_{1}}\left(x^{\prime} ; f\right) \\
& =O\left(\frac{A_{\nu}^{p-k_{1}}}{A_{\nu}^{p-k_{1}-1}} \frac{d^{i}}{d x^{j}} I_{n, \nu}^{p-k_{1}}\left(x^{\prime} ; f\right)\right) \\
& =O(\nu) \cdot O\left(\nu^{k 1}\right)=o\left(\nu^{k 1+1}\right) .
\end{aligned}
$$

This proves (14), given (13).

We shall also need the facts

$$
A_{p}^{p} \Delta^{p+1} \cos ^{p} \frac{\nu h}{2}=O\left(\frac{1}{n}\right)
$$

and

$$
A_{n \rightarrow p-k}^{p-k} \Delta^{p-k} \cos ^{p} \frac{n-p+k}{2} h=O\left(\frac{1}{n^{k}}\right) .
$$

These follow from the fact that $A_{\nu}^{p}=0\left(\nu^{p}\right)$ and that $\Delta^{p+1} \cos ^{p}$ $(\nu h / 2)=O\left(1 / n^{p+1}\right), \Delta^{p-k} \cos ^{p}(((n-p+k) / 2) h)=O\left(1 / n^{p}\right)$, the last two being consequences of the mean value theorem and the fact that $\lim _{n \rightarrow \infty}((n-p+k) / 2) h_{n}=\pi / 2$.

With this information we show that $d^{j} T_{n, p}\left(x^{\prime} ; f\right) / d x^{i} \rightarrow 0$ for $f$ of the prescribed type, by denoting in the expression (12) the two sums by $A$ and $B$, where

$$
\begin{aligned}
A & =\sum_{\nu=0}^{n-p-1} \frac{d^{i}}{d x^{j}} I_{n, \nu}^{p}\left(x^{\prime} ; f\right) A_{\nu}^{p}, \Delta^{p+1} \cos ^{p} \frac{\nu h}{2} \\
& =\sum_{v=0}^{n-p-1} o(1) \cdot O\left(\frac{1}{n}\right)=o(1), \quad \text { by (13) and (15), }
\end{aligned}
$$


and where

$$
\begin{array}{rlrl}
B & =\sum_{k=0}^{p} \frac{d^{j}}{d x^{j}} I_{n, n-p+k}^{p \rightarrow k}\left(x^{\prime} ; f\right) A_{n \rightarrow p+k}^{p \rightarrow k} \Delta^{p-k} \cos ^{p} \frac{n-p+k}{2} h \\
& =\sum_{k=0}^{p} o\left(n^{k}\right) \cdot O\left(\frac{1}{n^{k}}\right)=o(1), & & \text { by (14) and (16). }
\end{array}
$$

We see therefore that our theorem will be proved if (13) is established, and we now proceed to that.

We note first that

$$
I_{n, \nu}^{p}(x ; f)=\frac{1}{\pi} \int_{0}^{2 \pi} f(t) K_{\nu}^{p}(x-t) d \omega_{2 n+1}(t),
$$

where $K_{v}^{p}(u)$ is the pth Cesaro mean of order $\nu$ of the Dirichlet kernel $D_{n}(u)$. Thus it is clear that

$$
\begin{aligned}
\frac{d^{i}}{d x^{j}} I_{n, \nu}^{p}\left(x^{\prime} ; f\right) & =\frac{1}{\pi} \int_{0}^{2 \pi} f(t) \frac{d^{i}}{d x^{j}} K_{p}^{p}(x-t) d \omega_{2 n+1}(t) \\
& =\frac{1}{\pi} \int_{-\pi}^{\pi} f\left(x^{\prime}+t\right) \frac{d^{j}}{d t^{j}} K_{\nu}^{p}(t) d \omega_{2 n+1}\left(x^{\prime}+t\right) .
\end{aligned}
$$

Hence we have

$$
\left|\frac{d^{j}}{d x^{j}} I_{n, \nu}^{p}\left(x^{\prime} ; f\right)\right| \leqq \frac{1}{\pi} \int_{-\pi}^{\pi} \epsilon_{t}|t|^{j} \cdot\left|\frac{d^{j}}{d t^{j}} K_{\nu}^{p}(t)\right| d \omega_{2 n+1}\left(x^{\prime}+t\right),
$$

where $\epsilon_{t}$ is non-negative and approaches zero with $t$.

It is shown in Zygmund, Trigonometrical series, p. 259, that

$$
\left|\frac{d^{j}}{d t^{j}} K_{\nu}^{p}(t)\right| \leqq C \nu^{j+1}
$$

and that $\left|d^{i} K_{\nu}^{p}(t) / d t^{i}\right|$ is less than the sum of the three expressions

$$
C_{1} \sum_{k=1}^{s} \frac{\nu^{-k}}{|t|^{i+1+k}}, \quad C_{2} \sum_{\mu=0}^{j} \frac{\nu^{\mu-p}}{|t|^{p+1+j-\mu}}, \quad C_{3} \sum_{\mu=0}^{j} \frac{\nu^{-s+\mu}}{|t|^{s+j-\mu+1}},
$$

where the $C$ 's are constants, $s$ is any integer greater than $p+j$, and $|t| \leqq \pi$. It follows easily from this fact that

$$
|t|^{j} \cdot\left|\frac{d^{j}}{d t^{j}} K_{\nu}^{p}(t)\right| \leqq C \frac{1}{\nu t^{2}} \quad \text { for } \frac{1}{\nu} \leqq|t| \leqq \pi .
$$

We now use (18) and (19) to prove 


$$
\frac{1}{\pi} \int_{-\pi}^{\pi}|t|^{j} \cdot\left|\frac{d^{j}}{d t^{j}} K_{\nu}^{p}(t)\right| d \omega_{2 n+1}\left(x^{\prime}+t\right) \leqq C
$$

by splitting the integral in (20) into two parts, $\int_{-1 / \nu}^{1 / \nu}+\int_{R}=A+B$, where $R$ is the complement of $[-1 / \nu, 1 / \nu]$ in $[-\pi, \pi]$.

$$
\begin{aligned}
A & =\frac{1}{\pi} \int_{-1 / \nu}^{1 / \nu}|t|^{j}\left|\frac{d^{j}}{d t^{j}} K_{\nu}^{p}(t)\right| d \omega_{2 n+1}\left(x^{\prime}+t\right) \\
& \leqq C \nu^{j+1} \int_{-1 / \nu}^{1 / \nu}|t|^{j} d \omega_{2 n+1}\left(x^{\prime}+t\right) .
\end{aligned}
$$

Hence

$$
\begin{aligned}
A & \leqq C \nu^{j+1} \cdot \frac{1}{\nu^{j}} \int_{-1 / \nu}^{1 / \nu} d \omega_{2 n+1}\left(x^{\prime}+t\right) \\
& =C \nu \cdot \frac{2 \pi}{2 n+1} \cdot \frac{2 n+1}{2 \pi \nu}=C .
\end{aligned}
$$

Furthermore

$$
B=\frac{1}{\pi} \int_{R}|t|^{j} \cdot\left|\frac{d^{j}}{d t^{j}} K_{\nu}^{p}(t)\right| d \omega_{2 n+1}\left(x^{\prime}+t\right),
$$

so that by (19),

$$
B \leqq C \int_{R} \frac{1}{\nu t^{2}} d \omega_{2 n+1}\left(x^{\prime}+t\right)=C \frac{1}{\nu} \sum_{x_{k} \in R} \frac{1}{\left(x^{\prime}+x_{k}\right)^{2}} \cdot \frac{2 \pi}{2 n+1} .
$$

Now

$$
\begin{aligned}
\sum_{x_{k} \in R} \frac{1}{\left(x^{\prime}+x_{k}\right)^{2}} & \leqq 2 \sum_{j=0}^{\infty} \frac{1}{(1 / \nu+(2 \pi /(2 n+1)) j)^{2}} \\
& =2\left[\nu^{2}+\sum_{j=1}^{\infty} \frac{1}{(1 / \nu+(2 \pi /(2 n+1)) j)^{2}}\right] \\
& \leqq 2\left[\nu^{2}+\frac{2 n+1}{2 \pi} \int_{0}^{\infty} \frac{d u}{(1 / \nu+u)^{2}}\right] \\
& =2\left[\nu^{2}+\frac{2 n+1}{2 \pi} \nu\right] .
\end{aligned}
$$

Thus

$$
B \leqq C \frac{1}{\nu} \cdot \frac{2 \pi}{2 n+1} \cdot 2\left[\nu^{2}+\frac{2 n+1}{2 \pi} \nu\right] \leqq C^{\prime}
$$


and $(20)$ is proved.

To see that the integral in (17) can be made arbitrarily small by taking $\nu$ sufficiently large, we choose a $\delta>0$ small enough so that $\epsilon_{t}<\epsilon$ for $|t|<\delta$, and split the integral into two parts $\int_{-\delta}^{\delta}+\int_{S}=A+B, S$ being the complement of $[-\delta, \delta]$ in $[-\pi, \pi]$. Since by (19), $|t|^{i}\left|d^{i} K_{\nu}^{p}(t) / d t^{i}\right|$ approaches zero uniformly in $S$, we see that $B=o(1)$ as $\nu \rightarrow \infty$. Furthermore

$$
\begin{aligned}
A & =\frac{1}{\pi} \int_{-\delta}^{\delta} \epsilon_{t}|t|^{j} \cdot\left|\frac{d^{j}}{d t^{j}} K_{\nu}^{p}(t)\right| d \omega_{2 n+1}\left(x^{\prime}+t\right) \\
& \leqq \frac{\epsilon}{\pi} \int_{-\pi}^{\pi}|t|^{j}\left|\frac{d^{i}}{d t^{i}} K_{\nu}^{p}(t)\right| d \omega_{2 n+1}\left(x^{\prime}+t\right) \leqq \epsilon C, \quad \text { by }(20) .
\end{aligned}
$$

Thus $A+B$ can be made arbitrarily small by choosing $\nu$ sufficiently large.

Thus (13) is established and our theorem is proved.

It is not difficult to see that if $f$ has a $j$ th derivative $(j<p)$ at each point of an interval $a \leqq x \leqq b$ and that derivative is bounded (continuous) there, then the expression under the limit sign in (7) is uniformly bounded (convergent) in that interval.

It should be noted that a slight alteration in the proof of (20) yields the fact that if $f$ has a $p$ th derivative at the point $x^{\prime}$, then

$$
\frac{d^{p}}{d x^{p}} T_{n, p}\left(x^{\prime} ; f\right)=o(\log n) .
$$

University of Chicago 